\title{
Effect of Long-Term Use of Hormones on Endometriomas
}

\author{
Nicola Berlanda • Martina Morini • Dhouha Dridi • \\ Lucrezia de Braud • Benedetta Bracco • Paolo Vercellini
}

Published online: 12 July 2013

(C) Springer Science+Business Media New York 2013

\begin{abstract}
Surgery remains the standard treatment for endometriomas larger than $3 \mathrm{~cm}$. Three to six months of hormonal treatment administered preoperatively does not facilitate surgery; additionally, treatment taken postoperatively delays but does not prevent endometrioma recurrence and thus does not offer substantial long-term advantages. Nevertheless, postoperative long-term continuous use of oral contraceptives results in a $90 \%$ reduction in cyst recurrence compared with no treatment. The possible mechanisms of action of oral contraceptives in preventing endometrioma recurrence include hypomenorrhea, ovulation inhibition and improvement of progesterone resistance. Further studies are needed to assess whether long-term
\end{abstract}

\author{
N. Berlanda $\cdot$ M. Morini $\cdot$ D. Dridi $\cdot$ L. de Braud $\cdot$ B. Bracco $\cdot$ \\ P. Vercellini \\ "Luigi Mangiagalli" Department of Obstetrics and Gynecology, \\ Università degli Studi di Milano and Fondazione IRCCS Ca' \\ Granda Ospedale Maggiore Policlinico, Via della Commenda 12, \\ Milan, italy \\ M. Morini \\ e-mail: martina.morini19@gmail.com \\ D. Dridi \\ e-mail:dh.dridi2@gmail.com \\ L. de Braud \\ e-mail: ludebraud@hotmail.it \\ B. Bracco \\ e-mail: benedetta.bracco@studenti.unimi.it \\ P. Vercellini \\ e-mail: paolo.vercellini@unimi.it \\ P. Vercellini \\ Center for Research in Obstetrics and Gynecology (C.R.O.G.), \\ Viale Caldara 39, 20122 Milano, Italy

\section{N. Berlanda $(\square)$} \\ Clinica Ostetrica e Ginecologica "Luigi Mangiagalli”, Università \\ degli Studi di Milano and Fondazione IRCCS Ca' Granda Ospedale \\ Maggiore Policlinico, Via della Commenda 12, 20122 Milan, Italy \\ e-mail: nicola.berlanda@gmail.com
}

use of oral contraceptives might be effective in the treatment and prevention of endometriomas without surgery.

Keywords Endometrioma $\cdot$ Ovarian cysts $\cdot$ Laparoscopic surgery $\cdot$ Medical treatment of endometriosis · Oral contraceptives $\cdot$ Infertility $\cdot$ Pelvic pain

\section{Introduction}

During the last decades, the debate regarding the clinical management of endometriomas has focused more on surgical rather than medical treatment. During this period, the surgical approach has been modified from laparotomy to laparoscopy, a minimally invasive approach. Subsequently, laparoscopic techniques have been developed to reduce the surgical trauma to the healthy ovary. In this scenario, hormonal medical treatment has been administered either pre- or post- operatively for limited periods of time, i.e., 3 or 6 months, with the aim of improving surgical outcome. Unfortunately, however, these perioperative medical treatment regimens do not add any clinical advantages compared to surgical treatment alone. In 2004, in fact, a Cochrane review concluded that "there is no evidence of benefit associated with post-surgical medical therapy and insufficient evidence to determine whether there is a benefit from pre-surgical medical therapy" of endometriosis [1].

Recently, however, there has been a growing body of evidence suggesting that long-term hormonal treatment could play a central role in the postoperative management of patients with ovarian endometriotic cysts. The transition from 3 to 6 months to a long-term hormonal treatment for endometriomas is not only a practical matter of prolonging the time of drug administration, but it implies a conceptual change in the overall strategy for the management of this condition.

In the present paper, we review the available evidence on the hormonal treatment of endometriomas, with the aim of 
describing previous indications, the current treatment standard, and the possible future trends.

\section{Epidemiological and Clinical Characteristics of Endometriomas}

Endometriosis affects up to $10 \%$ of women of reproductive age [2]. The ovary is involved in more than half of the cases [3]. An ovarian endometrioma is a pseudocyst lined by endometrioid mucosa that contains a typical tarry, thick, chocolate-like fluid as the result of accumulation of hemorrhagic blood. Due to hemolysis of entrapped blood, chocolate fluid contains a very high iron concentration which creates an environment of a cytotoxic oxidative stress. Local high oxidative stress could be, at least in part, the mechanism of cellular toxicity responsible for either the reduction of the number of ovarian follicles, which may cause subfertility, or DNA damage, which may predispose to epithelial ovarian cancer $[4,5]$. Indeed epidemiologic studies demonstrated, in women with ovarian endometrioma, a higher incidence of endometrioid and clear cell carcinoma as compared to the general population, with an increased relative risk ranging between 1.3 and 2 [6].

At ultrasonography, chocolate fluid content has a typical homogeneous low echogenic pattern and therefore the sonographic diagnosis of endometriomas is highly reliable. Ovarian endometriotic cysts range in size from less than $1 \mathrm{~cm}$ up to 15 to $20 \mathrm{~cm}$. They may be asymptomatic or associated with pelvic pain. Endometriomas are unilateral in $70 \%$ and bilateral in $30 \%$ of cases; the left ovary is significantly more frequently affected than the right ovary with a $60 \%: 40 \%$ ratio [7].

\section{Pathogenesis of Endometriomas}

Three different pathogenic theories for endometriomas have been proposed [8]: 1) inversion and progressive invagination of the ovarian cortex after accumulation of menstrual debris deriving from bleeding of superficial endometriotic implants [9-11]; 2) secondary involvement of functional ovarian cysts by endometriotic implants located on the ovarian surface [12]; and 3) metaplasia of the coelomic epithelium covering the ovary [13]. The left-lateral predisposition of ovarian endometriotic cysts seems to support the first two "retrograde menstruation or transplantation" theories rather than the third "metaplasia" theory. In fact, the process of coelomic epithelium metaplasia would not be influenced by the pelvic anatomy. In contrast, the probability of peritoneal and ovarian implantation of endometrial cells refluxed through the fallopian tubes during menstruation may be higher in the left hemipelvis due to the presence of the sigmoid colon, which may create an anatomical shelter favouring the stasis of such cells and preventing them from being cleared by the circulating peritoneal fluid [7, 14].

Among the two "transplantation" theories, the former one referring to an invagination of the ovarian cortex has been suggested on the basis of the histological finding that in $90 \%$ of cases the endometriotic wall consists of ovarian cortex, demonstrated by the presence of primordial follicles [11]. Scurry et al. [15] have subsequently confirmed these findings; at histological evaluation of the endometrioma capsule, they identified two types of endometriotic cysts: one with endometrial lining, fibrosis, and ovarian cortical tissue, i.e. the cortical invagination endometrioma, in $42 \%$ of cases, and one with only endometrial lining and fibrosis in $58 \%$ of cases. These estimates are consistent with those reported in another study on the histology of the endometrioma wall [16], in which endometriosis, fibrosis, and ovarian cortical tissue were present in $46 \%$ of the specimens, whereas endometriosis and fibrosis were found in $54 \%$ of the specimens.

The second "implantation" theory postulates that an endometrioma develops from a functional ovarian cyst that undergoes invasion with endometriotic cells; in turn, these cells bleed inside the cyst and, eventually, the functional wall of the cyst gradually changes to become an endometrioma. Although a definitive histologic confirmation of this theory, i.e. a cyst with continuity between a functional cyst and endometrial lining, has never been observed [17], this theory regarding the origin of an endometrioma from a physiologic cyst is supported by ultrasonographic evidence; using serial transvaginal ultrasonographic evaluations, an endometrioma has been observed to develop from either an ovarian follicle [18] or a corpus luteum [19] (Fig. 1). Moreover, both surgical and histological evidence suggests that hemorrhagic functional cysts may play a role in the genesis of endometriosis-related chocolate cysts: first, chocolate-coloured fluid in absence of active endometriosis tissue has been laparoscopically demonstrated in the ovary, three months after drainage of an endometrioma in the same location followed by administration of GnRH agonist therapy [20]; and second, of 42 chocolate cysts diagnosed as endometrioma at the time of laparoscopy, only $68 \%$ were confirmed by pathology, with the other cases diagnosed as corpora lutea $(27 \%)$ or follicular cysts $(5 \%)$ [21].

\section{Indications and Limitations of Surgical Treatment of Endometriomas}

For infertile women, laparoscopic ovarian cystectomy sometimes may be recommended for endometriomas larger than $3 \mathrm{~cm}$ in diameter, because endometrioma fluid may damage the ovary and complications like peritonitis can arise in women with endometriomas undergoing ART [22]. Additional indications for surgery include the coexistence of unbearable 

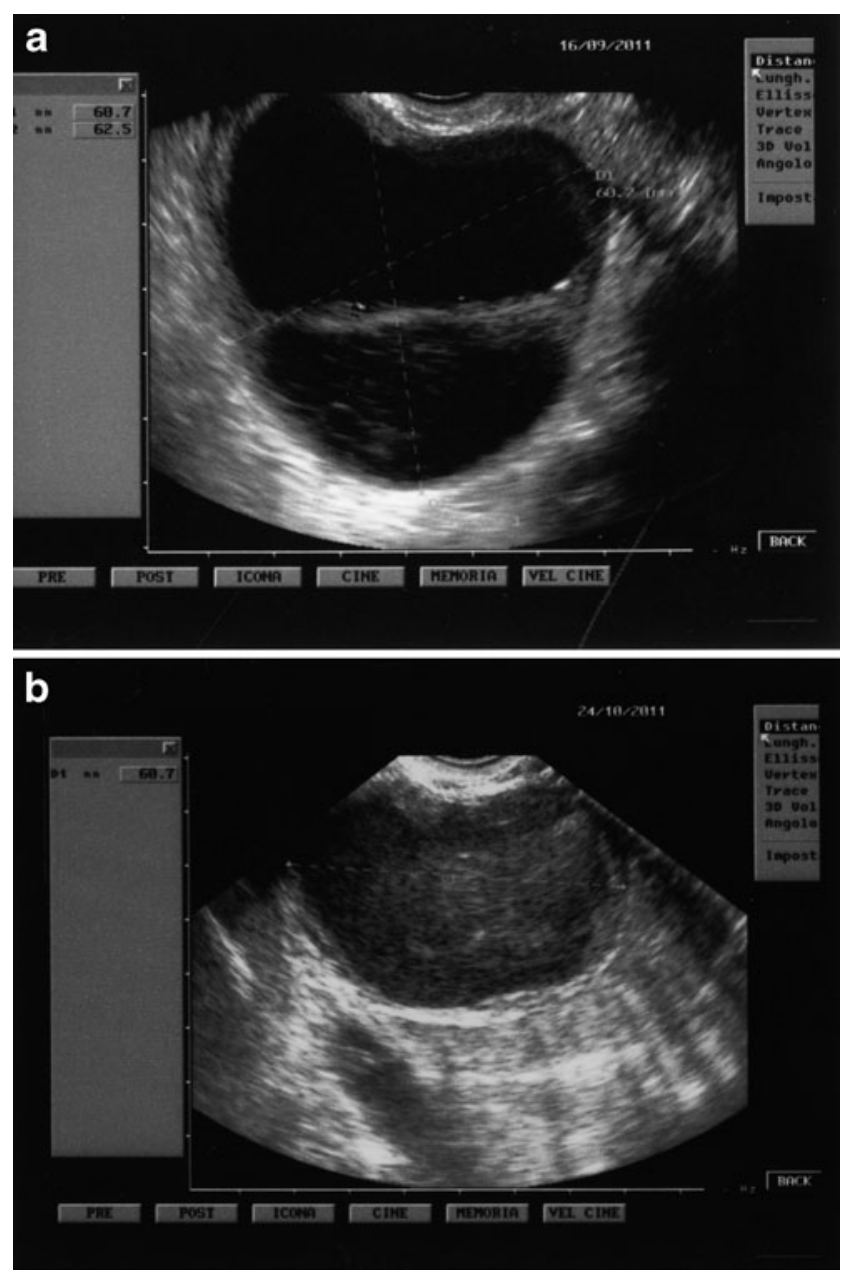

Fig. 1 a Cystic corpus luteum with reticular appearance in a previously normal ovary. b Same case as in 1A after 5 weeks. Progression of the cystic corpus luteum to an endometrioma, with homogeneous low echogenic fluid content

chronic pelvic pain, a rapid growth in endometrioma size, and the uncertainty regarding the precise nature of the cyst [23].

The standard surgical technique for endometrioma removal is laparoscopic stripping, that is, the gentle traction and countertraction of the pseudocystic wall (ovarian cortex surrounding so-called chocolate fluid) and the residual ovarian parenchyma by means of atraumatic forceps. Hemostasis of the pseudocystic bed is then achieved by pinpoint bipolar electrocoagulation [23]. An alternative technique consists of the fenestration and drainage of the endometrioma followed by laser vaporization (or bipolar coagulation) of the internal endometriotic foci. The results of a randomized, controlled trial (RCT) demonstrated a less severe effect on ovarian reserve, as defined by less diminished serum anti-Müllerian hormone levels in women who underwent the fenestrationvaporization technique as compared with the stripping technique [24]. However, data from two RCTs documented a substantial advantage of stripping over fenestration-vaporization in terms of spontaneous pregnancy rate and risk of endometrioma recurrence $[25,26]$.

In recent years, several studies have demonstrated that surgical excision of endometriomas is associated with a high rate of postoperative recurrences, ranging from $30 \%$ to $50 \%$ after 2 to 5 years [27-29]. Moreover, ultrasonographic evaluation of 93 ovaries in women undergoing IVF after the stripping of a unilateral endometrioma has shown that, despite the use of microsurgical and minimally invasive techniques, approximately $50 \%$ of operated ovaries show a reduced responsiveness to ovarian stimulation, and in approximately $13 \%$ of ovaries, a complete absence of follicular growth is observed, confirming severe impairment of ovarian reserve [30].

\section{Rationale and Available Compounds for the Hormonal Treatment of Endometriomas}

Because peritoneal and ovarian endometriosis is believed to arise from the implantation of regurgitated endometrial cells, the medical treatment strategies include the creation of a hypoestrogenic hormonal milieu to induce atrophy of both eutopic and ectopic endometrium, and to reduce or eliminate menstrual bleeding.

In light of the possible relationship between hemorrhagic functional ovarian cysts and the formation of endometriomas, suppression of ovulation might be particularly effective in the prevention of ovarian endometriosis [19]. Thus, whereas anovulation would act indirectly on endometriotic peritoneal implants, through the induction of a hypoestrogenic state and the reduction of menstrual bleeding, anovulation would directly inhibit the formation of functional cyst-related endometriomas.

In endometriosis, the eutopic and ectopic endometrium may not respond normally to progesterone and is considered by some investigators to be progesterone-resistant, which could contribute to proliferation and survival of implants [31]. One theory is that progesterone resistance in patients with endometriosis may be due to the over-expression of progesterone receptor A (PR-A) and the downregulation of progesterone receptor B (PR-B). In a recent paper, endometrioma samples obtained from patients treated with the progestin dienogest showed a significantly higher expression of PR-B compared with that observed in the control endometrioma samples. Dienogest treatment did not alter the expression of PR-A and thus the PR-B/PR-A ratio was improved [32].

The compounds most widely used for the treatment of endometriosis include danazol, gonadotrophin releasing hormone analogues (GnRH-a), progestogens, and the oral contraceptive pill (OC). These medications, unlike surgical excision, do not affect ovarian reserve.

The efficacy of these medications lasts only for as long as they are administered and, at treatment suspension, ovulatory 
cycles and menstrual bleeding are restored terminating the potential beneficial effects of treatment on endometriosis. Therefore, in order to maximize the preservation of ovarian reserve, long-term medical treatment, possibly until women seek conception, would be required.

Among the two most frequently used medications for the treatment of endometriosis, GnRH and OC, neither used as postoperative medical therapy has been shown to be superior in reducing the recurrence of endometriomas [31]. However, significant side effects and high costs limit treatment with GnRH-a to no more than 6 months. By contrast, OCs, together with progestins, offer a practical combination of benefits, risks, and costs [33-35], and based on the extensive epidemiologic information available, represent the safest medical alternatives for long-term treatment of endometriosis [36-38].

\section{Effects of Hormones on Endometriomas}

In a previous study evaluating the effect of ovarian stimulation on endometrioma size, 35 women with 45 endometriotic cysts underwent transvaginal ultrasonography the month preceding an IVF attempt and 3 to 6 months after the cycle. The median (interquartile range) diameter of endometriomas before and after the IVF cycle was unchanged: 20 (12-27) $\mathrm{mm}$ and 20 (17-27) mm, respectively, and new endometriomas were not detected [39]. More data are needed to confirm that ovarian stimulation affects neither the size of existing endometriomas nor formation of new endometriotic cysts.

The effect of ovarian suppression on existing endometriomas has been reported in six studies, evaluating the possible benefits of 3 to 6 months of hormonal treatment before surgical excision of the cysts. All six studies included a GnRH-a treatment group, which was compared to a notreatment group in four studies, to a danazol treatment group in one, and to both a no treatment and a danazol group in one.

In three studies, hormonal treatment was administered between an initial surgical procedure consisting in laparoscopic cyst drainage and a second surgical procedure consisting in laparoscopic or laparotomic second look/cyst excision. Two of these three studies were randomized, parallel group trials [40, $41]$; in both of them, the second surgical evaluation documented a significant reduction in endometrioma cyst size after 12weeks of treatment with GnRH-a compared with no treatment. The third study [42] demonstrated a $51 \%$ reduction in endometrioma size after administration of either GnRH-a or danazol for 6 months. In another study, follow-up evaluation was performed by means of transvaginal ultrasonography. In this study, two groups of women with endometriomas of mean diameter $4.5 \mathrm{~cm}$ (range, 2-7) were given either GnRH-a therapy for 3 months or no treatment after laparoscopic cyst drainage. Six months after the initial surgery, transvaginal ultrasound showed recurrent cysts in all women. There were no significant differences in mean endometrioma diameter between the two groups or between pre-laparoscopic and 6-month ultrasound examinations within each treatment group [43].

Two studies have compared the intraoperative and postoperative outcome of the laparoscopic excision of ovarian endometriotic cysts with and without preoperative hormonal treatment, without preliminary laparoscopic drainage. In one study, no significant difference was found between the two groups in total operative time, cyst excision time, complexity of surgery, and recurrence rates among patients who received a 3-month preoperative treatment with GnRH-a compared with a no-treatment group [44]. In the other study [45], patients with ovarian endometrioma undergoing a laparoscopic cystectomy were divided into three groups: no preoperative hormonal therapy; preoperative GnRH-a therapy for 3 to 6 months; and preoperative danazol therapy for 3 to 6 months. Mean diameter of endometriomas was reduced by preoperative hormonal therapy in both the GnRH-a and danazol groups. Interestingly, this study considered the effect of preoperative hormonal therapy on the histological characteristics of the endometrioma capsule. In this study, the preoperative hormonal therapy groups showed fibrosis in a higher number of capsules, with a significantly longer mean operation time compared with the no-therapy group; the loss of ovarian follicles was similar among the no therapy and hormone groups. The authors concluded that preoperative hormonal therapy caused severe fibrosis, which hampered stripping the cyst wall from the ovary and resulted in inadvertent removal of normal ovarian stroma.

The same authors have previously suggested [46] that when the endometrioma wall is attached to the ovarian stroma, the removal of the capsule is easy for the surgeon, but it is associated with damage to the ovarian stroma and loss of follicles. In contrast, stripping the capsule from invaginated cortex (in endometriomas that arise that way) does not affect the ovarian stroma or follicles. Similar considerations were reported by Nezhat et al. [9, 17], who proposed categorizing endometriomas based on their clinical characteristics arising from the corresponding purported pathogenic mechanism. According to them, type I endometriomas, i.e., the cortical invagination endometriomas, usually are $<5 \mathrm{~cm}$ in size, contain a dark fluid, and have capsules that are difficult to remove because they are associated with dense fibrosis and adhesions. Conversely, type II endometriomas, originating from functional cysts that were invaded by plaques of endometriosis, are larger in size and have a capsule that is more easily removed because of a clearer cleavage plane. More studies are needed to investigate the relationship among the different pathogenic, anatomical, and intraoperative characteristics of ovarian endometriotic cysts in relation to treatment paradigms in order to develop optimal approaches to treatment.

In conclusion, preoperative hormonal treatment of ovarian endometriomas has been associated with conflicting 
results. The most frequently reported effect has been a reduction of the cyst size, but this effect did not facilitate surgery nor did it improve surgical outcome; on the contrary, one study reported increased fibrosis of the cystic capsule associated with increased surgical difficulty.

However, the reduction in size observed with three to six months of medical therapy makes it tempting to speculate that a longer hormonal treatment might reduce endometrioma size even further. If this observation is valid, the use of primary hormonal treatment for small to medium-sized endometriomas (diameter $\leq 5 \mathrm{~cm}$ ) could be investigated with periodic ultrasonographic evaluation of the cyst size to confirm treatment benefit. In young, asymptomatic patients who wish to delay pregnancy for many years, postponing surgery until pregnancy is desired would be advantageous, to minimize the risks of surgical compromise of ovarian reserve.

\section{Effect of Postoperative Hormonal Treatment}

Considering the limitations of surgical excision of endometriomas, namely the high postoperative recurrence rate and the potential detrimental effect on ovarian reserve, the efficacy of ovarian suppression in prolonging the recurrence-free interval after laparoscopic excision of ovarian endometriomas has been evaluated.

\section{"Short-Term" 3 to 6 Months Treatment}

In three studies, the long-term recurrence rate of endometriomas was assessed among women who received a 3 to 6 months postoperative hormonal treatment compared with women who received no treatment. In one study [47], patients were randomized after surgery into four groups: placebo versus GnRH-a, continuous OCs, or dietary therapy for 6 months. Dietary therapy consisted of additional nutritional intake of vitamins (B6, A, $\mathrm{C}, \mathrm{E})$, mineral salts $(\mathrm{Ca}, \mathrm{Mg}, \mathrm{Se}, \mathrm{Zn}, \mathrm{Fe})$, lactic ferments, omega-3, and omega-6 fatty acids (fish oil). No statistically significant difference in endometrioma recurrence rate, as assessed by transvaginal ultrasonography, was detected 18months after surgery among any of the postoperative treatment groups compared with the placebo group. In another study [48], at a follow-up evaluation 36 months after surgery, the postoperative use of a $\mathrm{GnRH}$ agonist for 3 months delayed the time to recurrence by 2 months, and its use for 4 or 6 months delayed recurrence for approximately 5 months compared with the expectant management group. Despite such delay, time to recurrence was not significantly different among treated and untreated women. Similarly, a randomized, controlled trial reported that postoperative administration of OC for 6 months did not significantly affect the recurrence rate of endometriomas after a mean follow-up of 22 months (range 12-48) from surgical treatment. However, in this trial, the mean time to recurrence of endometriomas was delayed in the treatment group (18.2 months) compared with the control group (12.7months) [49].

Overall, in the former three studies, 137 women received GnRH-a and 134 received OC. Based on this limited amount of data, a 3- to 6-month course of postoperative hormonal treatment with either GnRH-a or OC does not significantly reduce the long-term recurrence risk of endometriomas.

\section{Long-Term Treatment}

In order to verify the hypothesis that the lack of efficacy of short-term postoperative treatment in preventing long-term recurrence of endometriomas could be caused by the rapid return to a regular follicular and hormonal activity after discontinuation of ovarian-suppressing hormones, some authors have evaluated the efficacy of a long-term treatment in reducing postoperative endometrioma recurrence.

Recently, a systematic MEDLINE search was conducted to identify all comparative studies published between January 2000 and February 2012 in the English language on the relationship between long-term postoperative adjuvant therapy and risk of endometrioma recurrence [50॰]. Of the 12 articles assessed in detail, 4 were selected based on surgery for endometriotic cysts, postoperative medical treatment use for $\geq 12$ months versus expectant management, and ultrasonographic and/or histological diagnosis of endometrioma recurrence. A total of 965 women were enrolled, 726 of whom were in three cohort studies [51-53] and 239 in one randomized controlled trial [54]. Oral contraceptives (OCs) were always used as the postoperative adjuvant treatment. The characteristics and the results of the four selected studies are reported in Table 1. As shown, the duration of OC use varied from $24[52,54]$ to 35 months [53]. Overall, an endometrioma recurrence was identified in 33 of 423 women ( $8 \%$; $95 \%$ confidence interval (CI) $6-11 \%$ ) in the OC group and in 117 of 341 women ( $34 \%$; $95 \%$ CI 29-40\%) in the no-treatment group. The overall OR of the four studies was 0.12 (95\% CI 0.05-0.29), meaning that the risk of endometrioma recurrence after first line surgery is reduced by $88 \%$ among OC users compared with non-OC users. In addition, among the three cohort studies, the endometrioma recurrence rate was compared between the 275 women who took the OC for the entire duration of follow-up, i.e., "always" users and the 179 women who took the OC discontinuously or for only part of the study period, i.e., "ever" users. In the former group, 16 endometriotic cysts were detected (6\%; $95 \%$ CI 4-9\%) compared with 48 in the latter (27\%; $95 \%$ CI $21-34 \%)$. The pooled OR was 0.21 (95\% CI 0.11-0.4). In other words, regular OC use beginning immediately after surgery was associated with only one-fifth of the risk of cyst recurrence observed in women who have used OC for short periods of time. Finally, when 
Table 1 Results of studies comparing the rate of recurrence of ovarian endometriotic cysts in women undergoing excision of endometriomas followed by oral contraceptive use for $\geq 2$ years versus no postoperative long-term medical treatment. Literature data, 2008-2010

\begin{tabular}{|c|c|c|c|c|c|c|c|}
\hline Authors, year & $\begin{array}{l}\text { Type of } \\
\text { study }\end{array}$ & $\begin{array}{l}\text { Number of } \\
\text { participants }\end{array}$ & $\begin{array}{l}\text { Postoperative } \\
\text { therapeutic scheme }\end{array}$ & $\begin{array}{l}\text { Months of } \\
\text { follow-up }\end{array}$ & $\begin{array}{l}\text { Analysis of } \\
\text { drop outs }\end{array}$ & $\begin{array}{l}\text { Recurrence in OC } \\
\text { group; } n(\%)\end{array}$ & $\begin{array}{l}\text { Recurrence in no } \\
\text { PLTT group; } n(\%)\end{array}$ \\
\hline $\begin{array}{l}\text { Vercellini et al., } \\
2008[51]\end{array}$ & Cohort & 277 & Cyclic OC vs. no PLTT & 28 & Yes & $9 / 102 *(9)$ & $26 / 46(56)$ \\
\hline $\begin{array}{l}\text { Takamura et al., } \\
2009 \text { [52] }\end{array}$ & Cohort & 87 & Cyclic OC vs. no PLTT & 24 & Yes & $1 / 34 *(3)$ & $17 / 39(44)$ \\
\hline $\begin{array}{l}\text { Seracchioli } \\
\quad \text { et al., } 2010 \\
\text { [54] }\end{array}$ & $\mathrm{RCT}$ & 239 & $\begin{array}{l}\text { Cyclic and continuous OC } \\
\text { vs. no PLTT }\end{array}$ & 24 & No & $17 / 148 \dagger(11)$ & $20 / 69(29)$ \\
\hline $\begin{array}{l}\text { Lee et al., } 2010 \\
\text { [53] }\end{array}$ & Cohort & 362 & $\begin{array}{l}\text { GnRH-a followed by OC } \\
\text { Short-term GnRH-a only }\end{array}$ & 35 & Yes & $6 / 139 *(4)$ & $54 / 187$ (29) \\
\hline
\end{tabular}

OC, oral contraceptive; PLTT, postoperative long-term treatment; RCT, randomised control trial; GnRHa, gonadotropin-releasing hormone analog *Only "always OC users" are considered. †Cyclic and continuous OC users are considered together

Modified from: Vercellini et al. Acta Obstet Gynecol Scand 2013;92:8-16

comparing ever users of OC with never users of OC, the overall OR of endometrioma recurrence after surgery was 0.39 , meaning that even irregular OC use was associated with a $60 \%$ reduction in risk compared with no OC use.

The protection against recurrence of endometrioma has been observed among patients receiving OC continuously, therefore experiencing amenorrhea, as well as those with hypomenorrhea induced by cyclical OC use. The observation that a comparable therapeutic effect is achieved independently of the presence or absence of OC-induced menstruations suggests that reducing endometrial reflux through the fallopian tubes might not be the only therapeutic mechanism of OC in preventing endometrioma recurrence. Additional or alternative mechanisms of action may include: 1) ovulation inhibition per se may constitute a protective factor, because it prevents the formation of endometriomas from functional ovarian cysts; and 2). OC administration might improve the progesterone resistance associated with endometriosis.

Based on the results of this systematic review and metaanalysis, OC use after conservative surgery for ovarian endometriotic cysts appeared to decrease the risk of endometrioma recurrence dramatically compared with no treatment, especially in women who used the contraceptive pill regularly and for prolonged periods of time. After first-line surgery for ovarian endometriomas, women should be informed of the high risk of cyst recurrence if no further treatment is undertaken and, whenever childbearing is deferred, regular OC use until pregnancy is desired should be strongly considered.

\section{Medical Treatment for the Primary Prevention of Endometriomas}

Based upon the strong protective effect of OC on both endometrioma recurrence and the development of functional cysts [55-57], OC use might be a strategy for endometrioma prevention. Unfortunately, epidemiological data are not available to support this approach. However, in a population at higher risk of developing endometriomas, such as first-degree relatives of women with endometriosis and endometriomas, the primary prevention of ovarian endometriotic cysts might prove cost-effective.

Epidemiological data were recently systematically reviewed regarding the relationship between OC exposure and risk of endometriosis [58], suggesting a reduction in risk of endometriosis in current $\mathrm{OC}$ users and a trend towards an increased risk in past $\mathrm{OC}$ users. However, the interpretation of these findings is difficult because the definitive confirmation or exclusion of endometriosis requires surgery, therefore, the exact point in time that endometriosis has occurred is not known. In particular, it is probable that the observed increase of endometriosis risk among past $\mathrm{OC}$ users simply reflects a delayed diagnosis due to the recurrence of symptoms suppressed by pill use with its discontinuation. In this regard, a study specifically designed to assess the risk of ovarian endometriomas among OC users and non-OC users in a subset of women at increased risk of developing this condition could overcome this limitation, as the ultrasonographic diagnosis of ovarian endometrioma is highly reliable.

\section{Conclusions}

A limitation of conservative surgical treatment of ovarian endometriotic cysts is a high recurrence rate. This recurrence rate only partially reflects the surgeon's ability and the surgical technique because of the intrinsic biology of endometriomas. In other words, because the pathogenesis of endometriosis is very likely to be multifactorial, including biochemical, endocrine, and inflammatory factors, surgery cannot simultaneously affect all these biological variables and therefore, importantly, surgical eradication of all visible endometriotic lesions does not eradicate 
the disease. Another limitation of conservative surgical treatment of ovarian endometriotic cysts is the possible association with follicle loss, which might diminish the fertility potential of operated women. As a consequence the therapeutic approach, especially with regards to recurrent cysts, must be extremely cautious because, obviously, repeat surgery is more likely to increase gonadal damage than a single procedure.

Similar to surgical treatment, hormonal therapy of endometriosis, although it allows suppression of the activity of endometriotic implants, is not able to eradicate the disease. Indeed, until a drug with the ability to selectively destroy ectopic endometrial cells and preserve the eutopic endometrium is available, which does not seem to be probable in the near future, medical treatment of endometriosis necessarily will continue to imply the survival of ectopic implants. Accordingly, reappearance of symptoms at drug discontinuation is expected and must not be considered as a demonstration of inefficacy of therapy.

Given the potential intrinsic limitations of both surgical and medical treatment of endometriosis and the fact that endometriosis should be viewed as a chronic disease that requires a lifelong management, the adequate approach to the disease is to maximize the use of medical treatment and avoid repeated surgical procedures [59]. In more practical terms, maximizing the use of medical treatment means that hormones may be administered for years, and therefore the first choice treatment is OCs, which are effective, safe, and well tolerated. At present, the available evidence strongly support the fact that long-term postoperative OC use should be considered a real therapeutic advance for patients operated for endometriomas.

Additional RCTs would be useful to determine the precise protective effect of adjuvant OC use definitively. However, given the impressive effect consistently observed in all the comparative studies considered, planning further trials including a no-treatment group would pose major ethical problems. Future studies designed to evaluate possible additional indications for OC treatment in the management of endometriomas are needed. Of particular interest would be the long term administration of OC in: 1) asymptomatic young women with ultrasonographic diagnosis of small to medium-sized endometriomas, i.e., with a maximal diameter $\leq 5 \mathrm{~cm}$, to determine whether cyst size may be reduced and surgical treatment postponed until pregnancy is desired, thus minimizing the risk of recurrence, as long as ultrasonographic follow-up assessments are performed regularly during treatment, and 2) women without the diagnosis of endometrioma, who are at increased risk of developing an endometrioma due to family history or other factors, to evaluate a possible role of $\mathrm{OC}$ in endometriotic cyst prevention.

\section{Compliance with Ethics Guidelines}

Conflict of Interest Paolo Vercellini received travel/accommodations expenses covered or reimbursed from ASRM.
Nicola Berlanda, Martina Morini, Dhouha Dridi, Lucrezia de Braud, and Benedetta Bracco declare that they have no conflict of interest.

Human and Animal Rights and Informed Consent This article does not contain any studies with human or animal subjects performed by any of the authors.

\section{References}

Papers of particular interest, published recently, have been highlighted as:

- Of importance

1. Yap C, Furness S, Farquhar C. Pre and postoperative therapy for endometriosis surgery. Cochrane Database Syst Rev. 2004;3:CD003678.

2. Eskenazi B, Warner ML. Epidemiology of endometriosis. Obstet Gynecol Clin North Am. 1997;24:235-58.

3. Jenkins S, Olive DL, Haney AF. Endometriosis: pathogenetic implications of the anatomic distribution. Obstet Gynecol. 1986;67:335-8.

4. Yamaguchi K, Mandai M, Toyokuni S, et al. Contents of endometriotic cysts, especially the high concentration of free iron, are a possible cause of carcinogenesis in the cysts through the ironinduced persistent oxidative stress. Clin Cancer Res. 2008;14:32-40.

5. Vercellini P, Crosignani $P$, Somigliana E, et al. The 'incessant menstruation'hypothesis: a mechanistic ovarian cancer model with implications for prevention. Hum Reprod. 2011;26:2262-73.

6. Vercellini P, Parazzini F, Somigliana E, et al. The endometriosisovarian cancer connection: the case against preventive surgery. Fertil Steril. 2009;91:e37.

7. Vercellini P, Aimi G, De Giorgi O, et al. Is cystic ovarian endometriosis an asymmetric disease? Br J Obstet Gynaecol. 1998;105:1018-21.

8. Vigano P, Somigliana E, Gentilini D, et al. Back to the original question in endometriosis: implantation or metaplasia? J Endometriosis. 2009;1:1-8.

9. Nezhat C, Nezhat C, Allen CJ, et al. Clinical and histologic classification of endometriomas. Implications for a mechanism of pathogenesis. J Reprod Med. 1992;37:771-6.

10. Brosens IA, Van Baller P, Puttermens PJ, Deprest J. Reconstruction of the ovary containing large endometriomas by an extraovarian endosurgical technique. Fertil Steril. 1996;66:517-21.

11. Hughesdon PE. The structure of endometrial cysts of the ovary. J Obstet Gynaecol Br Emp. 1957;64:481-7.

12. Sampson JA. Peritoneal endometriosis due to menstrual dissemination of endometrial tissue into the peritoneal cavity. Am J Obstet Gynecol. 1927;14:422-69.

13. Nisolle M, Donnez J. Peritoneal endometriosis, ovarian endometriosis, and adenomyotic nodules of the rectovaginal septum are three different entities. Fertil Steril. 1997;68:585-96.

14. Al-Fozan H, Tulandi T. Left lateral predisposition of endometriosis and endometrioma. Obstet Gynecol. 2003;101:164-6.

15. Scurry J, Whitehead J, Healey M. Classification of ovarian endometriotic cysts. Int J Gynecol Pathol. 2001;20:147-54.

16. Muzii L, Bianchi A, Crocè $C$, et al. Laparoscopic excision of ovarian cysts: is the stripping technique a tissue-sparing procedure? Fertil Steril. 2002;77:609-14.

17. Nezhat C, Nezhat C, Seidman D, et al. An expert forum for the histology of endometriomas. Fertil Steril. 2007;88:1017-8.

18. Jain S, Dalton ME. Chocolate cyst from ovarian follicles. Fertil Steril. 1999;72:852-6. 
19. Vercellini P, Somigliana E, Vigano P, et al. 'Blood on the Tracks' from corpora lutea to endometriomas. BJOG. 2009;116:366-71.

20. Donnez J, Nisolle MM, Cananas RF, et al. Endometriosis: rationale for surgery. In: Brosens I, Donnez J, editors. The current status of endometriosis research and management. Carnforth, UK: Parthenon Publishing; 1993. p. 385-96.

21. Koninckx PR, Muyldermans M, Moerman P, et al. CA 125 concentrations in ovarian 'chocolate' cyst fluid can differentiate an endometriotic cyst from a cystic corpus luteum. Hum Reprod. 1992;7:1314-7.

22. Johnson NP, Hummelshoj L; for the World Endometriosis Society Montpellier Consortium: consensus on current management of endometriosis. Hum Reprod 2013, Epub ahead of print.

23. Berlanda N, Vercellini P, Somigliana E, et al. Role of surgery in endometriosis-associated subfertility. Semin Reprod Med. 2013;31:133-43.

24. Tsolakidis D, Pados G, Vavilis D, et al. The impact on ovarian reserve after laparoscopic ovarian cystectomy versus three-stage management in patients with endometriomas: a prospective randomized study. Fertil Steril. 2010;94:71-7.

25. Beretta P, Franchi M, Ghezzi F, et al. Randomized clinical trial of two laparoscopic treatments of endometriomas: cystectomy versus drainage and coagulation. Fertil Steril. 1998;70:1176-80.

26. Alborzi S, Momtahan M, Parsanezhad ME, et al. A prospective, randomized study comparing laparoscopic ovarian cystectomy versus fenestration and coagulation in patients with endometriomas. Fertil Steril. 2004;82:1633-7.

27. Evers JL, Dunselman GA, Land JA, Bouckaert PX. Is there a solution for recurrent endometriosis? Br J Clin Pract Suppl. 1991;72:45-50. discussion 51-53.

28. DeCherney AH. Endometriosis: recurrence and retreatment. Clin Ther. 1992;14:766-72. discussion 765.

29. Guo SW. Recurrence of endometriosis and its control. Hum Reprod Update. 2009;15:441-61.

30. Benaglia L, Somigliana E, Vighi V, et al. Rate of severe ovarian damage following surgery for endometriomas. Hum Reprod. 2010;25:678-82.

31. Giudice LC, Kao LC. Endometriosis. Lancet. 2004;364:1789-99.

32. Hayashi A, Tanabe A, Kawabe S, et al. Dienogest increases the progesterone receptor isoform $\mathrm{B} / \mathrm{A}$ ratio in patients with ovarian endometriosis. J Ovarian Res. 2012;5:31.

33. Davis LJ, Kennedy SS, Moore J, Prentice A. Modern combined oral contraceptives for pain with endometriosis. Cochrane Database Syst Rev. 2007;18:CD001019.

34. Vercellini P, Cortesi I, Crosignani PG. Progestins for symptomatic endometriosis: a critical analysis of the evidence. Modern Trends. Fertil Steril. 1997;68:393-401.

35. Vercellini P, Fedele L, Pietropaolo G, et al. Progestogens for endometriosis: forward to the past. Hum Reprod Update. 2003;9:387-96.

36. American College of Obstetricians and Gynecologists. Noncontraceptive uses of hormonal contraceptives. Obstet Gynecol. 2010;115:206-18.

37. Vercellini P, Crosignani PG, Somigliana E, et al. Waiting for Godot: a commonsense approach to the medical treatment of endometriosis. Hum Reprod. 2011;26:3-13.

38. Hannaford PC, Iversen L, Macfarlane TV, et al. Mortality among contraceptive pill users: cohort evidence from Royal College of General Practinioners' Oral Contraceptive Study. Br Med J. 2010;340:c927.

39. Benaglia L, Somigliana E, Santi G, et al. IVF and endometriosisrelated symptom progression: insights from a prospective study. Hum Reprod. 2011;26:2368-72.

40. Donnez J, Anaf V, Nisolle M, et al. Ovarian endometrial cysts: the role of gonadotropin-releasing hormone agonist and/or drainage. Fertil Steril. 1994;62:63-6.

41. Shaw R, Garry R, McMillan L, et al. A prospective randomized open study comparing goserelin (Zoladex) plus surgery and surgery alone in the management of ovarian endometriomas. Gynaecol Endosc. 2001;10:151-7.

42. Rana N, Thomas S, Rotman C, Dmowski WP. Decrease in the size of ovarian endometriomas during ovarian suppression in stage IV endometriosis. Role of preoperative medical treatment. J Reprod Med. 1996;41:384-92.

43. Vercellini P, Vendola N, Bocciolone L, et al. Laparoscopic aspiration of ovarian endometriomas. Effect with postoperative gonadotropin releasing hormone agonist treatment. J Reprod Med. 1992; 37:577-80.

44. Muzii L, Marana R, Caruana P, Mancuso S. The impact of preoperative gonadotropin-releasing hormone agonist treatment on laparoscopic excision of ovarian endometriotic cysts. Fertil Steril. 1996;65:1235-7.

45. Tsujioka $\mathrm{H}$, Inoue $\mathrm{Y}$, Emoto $\mathrm{M}$, et al. The efficacy of preoperative hormonal therapy before laparoscopic cystectomy of ovarian endometriomas. J Obstet Gynaecol Res. 2009;35:782-6.

46. Hachisuga T, Kawarabayashi T. Histopathological analysis of laparoscopically treated ovarian endometriotic cysts with special reference to loss of follicles. Hum Reprod. 2002;17:432-5.

47. Sesti F, Capozzolo T, Pietropolli A, et al. Recurrence rate of endometrioma after laparoscopic cystectomy: a comparative randomized trial between post-operative hormonal suppression treatment or dietary therapy vs. placebo. Eur J Obstet Gynecol Reprod Biol. 2009;147:72-7.

48. Jee BC, Lee JY, Suh CS, et al. Impact of GnRH agonist treatment on recurrence of ovarian endometriomas after conservative laparoscopic surgery. Fertil Steril. 2009;91:40-5.

49. Muzii L, Marana R, Caruana P, et al. Postoperative administration of monophasic combined oral contraceptives after laparoscopic treatment of ovarian endometriomas: a prospective, randomized trial. Am J Obstet Gynecol. 2000;183:588-92.

50. • Vercellini P, De Matteis S, Somigliana E, et al. Long-term adjuvant therapy for the prevention of postoperative endometrioma recurrence: a systematic review and meta-analysis. Acta Obstet Gynecol Scand. 2013;92:8-16. This study review available evidence demonstrating that postoperative long term hormonal treatment is effective in reducing the recurrence rate of endometriomas.

51. Vercellini P, Somigliana E, Daguati R, et al. Postoperative oral contraceptive exposure and risk of endometrioma recurrence. Am J Obstet Gynecol. 2008;198:504.e1-5.

52. Takamura M, Koga K, Osuga Y, et al. Post-operative oral contraceptive use reduces the risk of ovarian endometrioma recurrence after laparoscopic excision. Hum Reprod. 2009;24:3042-8.

53. Lee DY, Bae D, Yoon B, Choi D. Post-operative cyclic oral contraceptive use after gonadotrophin-releasing hormone agonist treatment effectively prevents endometrioma recurrence. Hum Reprod. 2010;25:3050-4.

54. Seracchioli R, Mabrouk M, Frascà $C$, et al. Long-term cyclic and continuous oral contraceptive therapy and endometrioma recurrence: a randomized controlled trial. Fertil Steril. 2010;93:52-6.

55. Ory H. Boston Collaborative Drug Surveillance Program: functional ovarian cysts and oral contraceptives. Negative association confirmed surgically. A cooperative study. J Am Med Assoc. 1974;228:68-9.

56. Vessey M, Metcalfe A, Wells C, et al. Ovarian neoplasms, functional ovarian cysts, and oral contraceptives. Br Med J. 1987;294:1518-20.

57. Booth M, Beral V, Maconochie N, et al. A case-control study of benign ovarian tumors. J Epidemiol Commun Health. 1992;46:528-31.

58. Vercellini P, Eskenazi B, Consonni D, et al. Oral contraceptives and risk of endometriosis: a systematic review and meta-analysis. Hum Reprod Updat. 2011;17:159-70.

59. Practice Committee of the American Society for Reproductive Medicine. Treatment of pelvic pain associated with endometriosis. Fertil Steril. 2008;90:S260-9. 